REVIEW

Acute liver failure

J G O'Grady

Postgrad Med J 2005;81:148-154. doi: 10.1136/pgmj.2004.026005

Acute liver failure is a complex multisystemic illness that evolves quickly after a catastrophic insult to the liver leading to the development of encephalopathy. The underlying aetiology and the pace of progression strongly influence the clinical course. The commonest causes are paracetamol, idiosyncratic drug reactions, hepatitis B, and seronegative hepatitis. The optimal care is multidisciplinary and up to half of the cases receive liver transplants, with survival rates around 75\%-90\%. Artificial liver support devices remain unproven in efficacy in acute liver failure.

Correspondence to: Dr J G O'Grady, Institute of Liver Studies, King's College Hospital, Denmark Hill, London SE5 9PJ, UK john.o'grady@kcl.ac.uk

Submitted 24 June 2004 Accepted 11 August 2004
A cute liver failure (ALF) is a complex multisystemic illness that evolves after a catastrophic insult to the liver manifesting in the development of a coagulopathy and encephalopathy within a short period of time. ALF is a heterogenous condition incorporating a range of clinical syndromes, principally determined by the underlying aetiology, the age of the patient, and the duration of time over which the disease evolves. There are a number of classifications of ALF in use but in the UK the terms hyperacute, acute, and subacute are used to define the onset of encephalopathy within 7 days, 8-28 days, and more than 28 days, respectively. ${ }^{1}$ The natural history of the condition is very variable within this range and survival rates without transplantation range from $10 \%$ to $90 \%$ for different cohorts. Integrated multidisciplinary protocols that use liver transplantation are now achieving considerably improved survival rates in the range from $40 \%$ to $90 \%$ depending on the underlying aetiology.

The components of the management strategy are:

- determination of aetiology

- assessment of the severity of the disease and the associated prognosis,

- prevention or treatment of complications

- liver transplantation when spontaneous survival is considered unlikely

- possible use of liver support devices.

\section{CAUSES AND EPIDEMIOLOGY}

There is considerable geographical variation in the causation of ALF (table 1). Viruses and drugs account for most cases, but a significant number of patients have no definable cause and are classified as seronegative or of being of indeterminate aetiology. Paracetamol is the commonest cause in the UK and USA. ${ }^{2}$ Idiosyncratic reactions comprise another important group.

Viral

ALF is an uncommon complication of viral hepatitis, occurring in $0.2 \%-4 \%$ of cases depending on the underlying aetiology. ${ }^{3}$ The risk is lowest with hepatitis A, but it increases with the age at time of exposure. Hepatitis $\mathrm{B}$ can be associated with ALF through a number of scenarios (table 2). The commonest are de novo infection and spontaneous surges in viral replication, while the incidence of the delta virus infection seems to be decreasing rapidly. Vaccination should reduce the incidence of hepatitis A and B, while antiviral drugs should ameliorate replication of hepatitis B. Hepatitis C is rarely recognised as the sole cause of ALF. Hepatitis E is common in parts of Asia and Africa and the risk of developing ALF increases to over $20 \%$ in pregnant women, being particularly high during the third trimester. Unusual causes of viral ALF include herpes simplex 1 and 2, herpesvirus-6, varicella zoster, Epstein-Barr virus, and cytomegalovirus.

Seronegative hepatitis is the commonest presumed viral cause in some parts of the western world, although there is little evidence to implicate a viral infection. Middle aged women are most commonly affected and it occurs sporadically. The diagnosis is one of exclusion.

\section{Drugs}

Paracetamol overdose accounts for about $40 \%$ of cases of ALF in the UK but is falling since legislation limited the amounts that can be purchased over the counter. ${ }^{2}$ It is usually taken with suicidal or parasuicidal intent, but up to $8 \%-30 \%$ cases are consequent upon therapeutic use. Factors increasing susceptibility to paracetamol toxicity include regular alcohol consumption, antiepileptic therapy (via enzyme induction), and malnutrition. ALF develops in only $2 \%-5 \%$ of those taking overdoses,. There is some dose dependent relation with mortality, which is highest at doses over 48 grams.

Idiosyncratic drug reactions usually develop during the first exposure to the drug and the leading candidates are listed in box 1 . The diagnosis is usually made on the basis of a temporal relation between exposure to the drug and the liver injury. Estimates of the risk of developing ALF as a result of an idiosyncratic reaction range from $0.001 \%$ for non-steroidal anti-inflammatory drugs to $1 \%$ 
Table 1 Geographical variation in the aetiology of ALF

\begin{tabular}{llllll}
\hline & UK & US & France & India & Japan \\
\hline Paracetamol & 54 & 40 & 2 & - & - \\
Drug reactions & 7 & 12 & 15 & 5 & - \\
Seronegative & 17 & 17 & 18 & 24 & 45 \\
Hepatitis A or B & 14 & 12 & 49 & 33 & 55 \\
Hepatitis E & - & - & - & 38 & - \\
Other causes & 8 & 19 & 16 & - & - \\
\hline \multicolumn{7}{l}{ Data shown as percentages. } \\
\end{tabular}

Table 2 ALF associated with hepatitis B

\begin{tabular}{|c|c|c|c|c|c|c|}
\hline & HbsAg & $\lg M$ anti-core & $\mathrm{HbeAg}$ & HbeAb & HBV DNA & Comment \\
\hline Acute infection & Variable & Positive & Variable & Variable & Usually negative & Hyperactive response, immune mediated \\
\hline Seroconversion & Positive & Negative & Negative & Positive & Negative & Immune \\
\hline Replication surge & Positive & Negative & Variable & Variable & High & Spontaneous or after immunsuppression \\
\hline Delta super-infection & Positive & Negative & Variable & Variable & Low & New positive serology for delta virus \\
\hline
\end{tabular}

for the isoniazid/rifampicin combination. Ecstasy (3, 4 methylenedioxymetamphetamine-a synthetic amphetamine) has been associated with a number of clinical syndromes ranging from rapidly progressive ALF associated with malignant hyperpyrexia to subacute liver failure.

\section{Box 1 Drugs causing ALF}

\section{Category 1-commoner causes}

- Paracetamol, halothane, isoniazid/rifampicin, nonsteroidal anti-inflammatory drugs (NSAIDs), sulphonamides, flutamide, sodium valproate, carbamazepine, Ecstasy

\section{Category 2-rarer causes}

- Benoxyprofen, phenytoin, isoflurane, enflurane, tetracycline, allopurinol, ketoconazole, monoamine oxidase inhibitors (MAOls), disulphiram, methyldopa, amiodarone, tricyclic antidepressants, propylthiouracil, gold, 2,3,-dideoxyinosine (ddl)

\section{Other aetiologies}

There are three recognised syndromes of liver injury that usually occur during the third trimester, although a considerable degree of overlap exists. Acute fatty liver of pregnancy preferentially affects primagravids carrying a male fetus. The HELLP syndrome is defined by the co-existenxce of haemolysis, elevated liver enzymes, low platelets. ALF complicating pre-eclampsia or eclampsia is characterised by high transaminase activities.

Wilson's disease may present as ALF, usually during the second decade of life and is characterised clinically by a Coomb's negative haemolytic anaemia and demonstrable Kayser-Fleischer rings in most cases. Poisoning with Amanita phalloides (mushrooms) is most commonly seen in central Europe, South Africa, and the west coast of the United States. Severe diarrhoea, often with vomiting, starts five or more hours after ingestion of the mushrooms and liver failure develops four to five days later. Autoimmune chronic hepatitis may present as ALF but in a condition that is no longer responsive to corticosteroid therapy or other immunosuppressive therapy.
The Budd-Chiari syndrome may present with ALF and the diagnosis is suggested by hepatomegaly and confirmed by the demonstration of hepatic vein thrombosis. Malignancy infiltration, especially with lymphoma, is also typically associated with hepatomegaly. Ischaemic hepatitis is being increasingly recognised as a cause of ALF, especially in older patients.

- Paracetamol remains the commonest aetiology but the incidence is falling in the UK

- Seronegative hepatitis is the next biggest group

- Hepatitis A and B should decrease with vaccination programmes

- Idiosyncratic drug reactions comprise the other main aetiological group

\section{DIAGNOSIS AND PROGNOSIS}

The aetiology of ALF must be accurately identified and the appropriate investigations are outlined in table 3. A liver biopsy is not usually necessary other than to identify the occasional case of malignant disease. The underlying aetiology is a powerful determinant of prognosis with a spontaneous survival rate ranging from $10 \%$ to $90 \%$. The grade of encephalopathy also correlates strongly with outcome (both the grade at presentation and the maximum grade attained). The prognosis deteriorates further when grade 4 encephalopathy is complicated by cerebral oedema, and even further when the latter coexists with renal failure. ${ }^{4}$

Reliance on the development of these clinical complications to determine prognosis is not helpful when defining the scope and application of liver transplantation and early indicators of prognosis are required. Furthermore, patients with subacute liver failure have very poor prognoses despite not developing cerebral oedema or renal failure. Determination of prognosis drives two fundamental management issues - that is, the need for referral to specialist centres and the indications for transplantation. Indications for referral to specialist units have been suggested for paracetamol and other aetiologies of ALF (tables 4, 5). Separate criteria have been identified for use within specialist centres to identify the 
Table 3 Investigation of aetilogy of ALF

\begin{tabular}{|c|c|}
\hline Aetiology & Investigation \\
\hline $\begin{array}{l}\text { Hepatitis A (HAV) } \\
\text { Hepatitis B + D (HBV, HDV) } \\
\text { Hepatitis E (BEV) } \\
\text { Paracetamol } \\
\text { Idiosyncratic drug reactions } \\
\text { Autoimmune } \\
\text { Pregnancy related syndromes } \\
\quad \text { fatty liver } \\
\text { HELLP syndrome } \\
\text { toxaemia } \\
\text { Wilson's disease } \\
\\
\text { Budd-Chiari syndrome } \\
\text { Malignancy } \\
\text { Ischaemic hepatitis }\end{array}$ & $\begin{array}{l}\text { IgM anti-HAV } \\
\text { HBsAg may be negative, IgM anti- } \\
\text { core, HBV DNA } \\
\text { anti-HEV } \\
\text { drug concentrations in blood } \\
\text { eosinophil count } \\
\text { autoantibodies, lgGs } \\
\text { ultrasound, uric acid, histology } \\
\text { platelet count } \\
\text { serum transaminases } \\
\text { urinary copper, ceruloplasmin, slit- } \\
\text { lamp examination } \\
\text { ultrasound or venography } \\
\text { imaging and histology } \\
\text { transaminases }\end{array}$ \\
\hline
\end{tabular}

cohort most in need of liver transplantation (tables 6,7$).{ }^{5}$ The paracetamol criteria have been supplemented by the use of serum lactate concentrations with readings above 3-3.5 being associated with a poor prognosis. ${ }^{6}$ These criteria were not validated in a number of rare aetiologies particularly pregnancy related syndromes (liver transplantation rarely required), Wilson's disease (liver transplantation always required), and Amanita phalloides poisoning.

- Early assessment of prognosis helps development of management strategies and need for liver transplantation

- Criteria have been described for referral to specialist centre

- Separate criteria have been described for selection of patients for liver transplantation
The prognostic value of histological examination is limited by sample variation and in subacute liver failure by the presence of nodules of regeneration that do not correlate with survival. A small liver on clinical or radiological assessment, or more particularly a liver that is found to be shrinking rapidly, is a poor prognostic indicator. This feature is especially useful in subacute liver failure when the degree of encephalopathy and the severity of the derangement of coagulation may not be particularly pronounced. Serial volumetric studies using computed tomography has been used to assess both the size of the liver and the functional reserve and this may be useful in determining prognosis.

\section{MANAGEMENT \\ Overall strategy}

Each patient with ALF needs an overall management plan that starts with identification of aetiology, rescusitation, and an initial assessment of prognosis. Appropriate patients should be referred to specialist centres offering liver transplantation. Patients are monitored for complications and these are treated as they emerge to the point of recovery, death, or transplantation. Patients not initially considered for transplantation may change status on the basis of prognostic indicators or the pattern of clinical complications that emerges. Likewise, patients listed for transplantation may develop complications that preclude this intervention or occasionally may show unexpected signs of recovery before a donor organ becomes available. The final decision on transplantation is made when an organ is available.

\section{Initial management}

Patients presenting with a history of paracetamol ingestion should be given $\mathrm{N}$-acetylcysteine intravenously pending the result of the paracetamol concentration in blood. This should then determine those who continue with treatment. The other mandatory blood tests are prothrombin time or INR, serum creatinine, and acid base status. Liver function tests are of limited value in quantifying the severity of the liver injury except at the milder end of the spectrum. Aggressive rehydration is advised, especially in those with acidosis or oliguria. Serial investigations should be interpreted as outlined in table 4.

Table 4 Referral to specialist unit after paracetamol ingestion. Any of these criteria should prompt referral

\begin{tabular}{lll}
\hline Day 2 & Day 3 & Day 4 \\
\hline Arterial $\mathrm{pH}<7.30$ & Arterial $\mathrm{pH}<7.30$ & INR $>6$ or PT $>100 \mathrm{~s}$ \\
$\mathrm{INR}>3.0$ or PT $>50 \mathrm{~s}$ & INR $>4.5$ or PT $>75 \mathrm{~s}$ & Progressive rise in PT to any level \\
Oliguria & Oliguria & Oliguria \\
Creatinine $>200 \mu \mathrm{mol} / /$ & Creatinine $>200 \mu \mathrm{mol} / \mathrm{I}$ & Creatinine $>300 \mu \mathrm{mol} / /$ \\
Hypoglycaemia & Encephalopathy & Encephalopathy \\
& Severe thrombocytopenia & Severe thrombocytopenia \\
\hline INR, international normalised ratio; PT, prothrombin time. & \\
\hline
\end{tabular}

Table 5 Referral to specialist unit in non-paracetamol aetiologies. The presence of any of the following criteria should prompt referral

\begin{tabular}{lll}
\hline Hyperacute & Acute & Subacute \\
\hline Encephalopathy & Encephalopathy & Encephalopathy \\
Hypoglycaemia & Hypoglycaemia & Hypoglycaemia (less common) \\
Prothrombin time $>30 \mathrm{~s}$ & Prothrombin time $>30 \mathrm{~s}$ & Prothrombin time $>20 \mathrm{~s}$ \\
INR $>2.0$ & INR $>2.0$ & INR $>1.5$ \\
Renal failure & Renal failure & Renal failure \\
Hyperpyrexia & & Serum sodium $<130 \mu \mathrm{mol} / \mathrm{I}$ \\
& & Shrinking liver volume \\
\hline INR, international normalised ratio. &
\end{tabular}


Table 6 Indicators of a poor prognosis in paracetamol induced ALF

\begin{tabular}{llll}
\hline Parameter & Sensitivity & Specificity & $\begin{array}{l}\text { Positive predictive } \\
\text { accuracy }\end{array}$ \\
\hline $\begin{array}{l}\text { Arterial } \mathrm{pH}<7.30^{*} \\
\text { All three of the following }\end{array}$ & 49 & 99 & 81 \\
concomitantly: prothrombin time $>100 \mathrm{~s}$ & 45 & 94 & 67 \\
$\begin{array}{l}\text { or INR }>6.5, \text { creatinine }>300 \mu \mathrm{mol} / \mathrm{I} \\
\text { and grade 3-4 encephalopathy }\end{array}$ & & \\
\hline Data shown as percentages. & & \\
\hline
\end{tabular}

Patients with other forms of liver disease should be treated symptomatically and rehydrated. The prothrombin time or INR is measured serially to monitor the severity of the liver injury. Lamivudine should be considered in patients with hepatitis B related disease. Penicillamine may be of value in patients with Wilson's disease who are not manifesting encephalopathy. Immunosuppression should be used judiciously in autoimmune hepatitis. Patients with severe hepatitis should be monitored regularly for hypoglycaemia. Sedatives and narcotic analgesics should be avoided.

\section{Encephalopathy}

The diagnosis of encephalopathy is central to the diagnosis of ALF and is graded from 1 to 4 depending on clinical severity (table 8). Cerebral oedema may complicate advanced encephalopathy. Patients with acute and hyperacute liver failure are at greater risk of developing grade IV coma and cerebral oedema.

The aetiological factors in the development of encephalopathy remain unclear but it is believed that there is a build up of putative toxins (ammonia, mercaptans, $\gamma$-aminobutyric acid, endogenous benzodiazepines, and serotonin/tryptophan), changed or false neurotransmittor levels, and changed receptor activation (NMDA) and GABAergic tone. ${ }^{7}$ The pathogenesis of cerebral oedema incorporates both increased blood flow with loss of autoregulation and astrocyte swelling. Inflammatory mediators are also of importance, as shown by the impressive stabilisation in neurological function that is often observed when the diseased liver is removed during the transplant operation.
Patients are usually mechanically ventilated once grade 3 encephalopathy develops. Sedation should be used to reduce stimuli that can aggravate cerebral oedema and minimise the risk of unrecognised seizure activity. The risk/benefit ratio of invasive intracranial pressure monitoring needs to be assessed for each patient. The benefit of knowing the precise pressures and the response to treatment need to be offset by the risk of haemorrhage and infection. ${ }^{89}$ Treatment is targeted to maintain intracranial pressures below $25 \mathrm{~mm}$ $\mathrm{Hg}$, cerebral perfusion pressures above $50 \mathrm{~mm} \mathrm{Hg}$, and jugular venous saturations between $55 \%-80 \%$. The treatment modalities include mannitol (mainstay of treatment), barbiturates (used less commonly than previously), hypothermia (experimental), hypertonic normal saline, vasopressors, hepatectomy, and liver transplantation. ${ }^{3}{ }^{10} 11$

\section{Cardiovascular and respiratory}

A hyperdynamic circulation with peripheral vasodilatation and central volume depletion leading to hypotension are characteristic of ALF. Hypotension may respond to volume repletion but many patients will require vasopressor therapy directed by invasive haemodynamic monitoring. It has recently been recognised that adrenal dysfunction can contribute to refractory hypotension and a trial of hydrocortisone is warranted in these patients. ${ }^{12}$

Sepsis, haemorrhage, pleural effusions, atelectasis, and intrapulmonary shunts may contribute to respiratory difficulty. However, most cases of severe lung injury have adult respiratory distress syndrome with or without pulmonary sepsis. Pulmonary sepsis should be aggressively sought and

Table 7 Indicators of a poor prognosis in non-paracetamol aeiologies of ALF

\begin{tabular}{|c|c|c|c|}
\hline Parameter & Sensitivity & Specificity & $\begin{array}{l}\text { Positive predictive } \\
\text { accuracy }\end{array}$ \\
\hline $\begin{array}{l}\text { Prothrombin time }>100 \mathrm{~s} \\
\text { Any three of following: unfavourable } \\
\text { aetiology (seronegative hepatitis or drug } \\
\text { reaction age }<10 \text { or }>40 \text { years, acute or } \\
\text { subacute categories, serum bilirubin }>300 \mu \mathrm{mol} / \mathrm{I} \text {, } \\
\text { prothrombin time }>50 \text { seconds or INR }>3.5\end{array}$ & $\begin{array}{l}34 \\
93\end{array}$ & $\begin{array}{l}100 \\
90\end{array}$ & $\begin{array}{l}46 \\
92\end{array}$ \\
\hline
\end{tabular}

Data shown as percentages.

Table 8 Modified Parsons-Smith scale of hepatic encephalopathy

\begin{tabular}{|c|c|c|c|}
\hline Grade & Clinical features & Neurological signs & $\begin{array}{l}\text { Glasgow coma } \\
\text { scale }\end{array}$ \\
\hline 0/subclinical & Normal & $\begin{array}{l}\text { Only seen on neuro- } \\
\text { psychometric testing }\end{array}$ & 15 \\
\hline 1 & $\begin{array}{l}\text { Trivial lack of awareness, shortened } \\
\text { attention span }\end{array}$ & $\begin{array}{l}\text { Tremor, apraxia, } \\
\text { incoordination }\end{array}$ & 15 \\
\hline 2 & Lethargy, disorientation, personality change & Asterixis, ataxia, dysarthria & $11-15$ \\
\hline 3 & $\begin{array}{l}\text { Confusion, somnolence to semi-stupor, } \\
\text { responsive to stimuli }\end{array}$ & Asterixis, ataxia & $8-11$ \\
\hline 4 & Coma & \pm decerebration & $<8$ \\
\hline
\end{tabular}


treated. Pleural effusions may require drainage if they are impeding ventilation. Severe hypoxaemia may respond to postural changes and pronation or inhalation of nitric oxide.

\section{Renal and metabolic}

The aetiology of renal dysfunction is a mixture of functional or hepatorenal failure and acute tubular necrosis. Renal failure is particularly common with paracetamol induced liver failure where the toxicity is a direct effect on the renal tubule. Adequate volume repletion and the avoidance of nephrotoxins are central to the maintenance of renal function. Enthusiasm for the use of dopamine has waned. Intra-abdominal hypertension may reduce renal perfusion pressure and the measurement of intra-abdominal pressure may be a valuable component of monitoring in selected cases.

Early renal replacement therapy is recommended in established renal failure. Continuous, rather than intermittent, modes of treatment are preferred to minimise the haemodynamic and cerebral fluctuations that may occur during the initiation of extracorporeal therapy. ${ }^{13}$ Inability of the liver to metabolise and use lactate or acetate buffer solutions means that bicarbonate buffers are preferred in this setting and these provide more effective control of acid base status. Anticoagulation is required despite the presence of a significant coagulopathy to minimise depletion of platelets and prevent the filters clotting. Patients with clinical manifestation of coagulopathy may benefit from epoprostenol, regional heparinisation, and citrate. A range of metabolic abnormalities may be seen and will often require appropriate attention (box 2).

\section{Box 2 Metabolic abnormalities in ALF independent of renal function}

- Hypoglycaemia

- Hyponatraemia

- Hypokalaemia

- Hypophosphataemia

- Metabolic alkalosis

\section{Sepsis}

Systemic inflammatory response syndromes are commonly seen in patients with ALF, sometimes in the absence of microbiologically confirmed infection. ${ }^{14}$ Established bacterial and fungal infections occur in about $80 \%$ and $32 \%$, respectively. These patients are functionally immunosuppressed with impaired cell mediated immunity, complement levels, and phagocytosis. Meticulous care with hygiene and prevention of nosocomial infection are impertative. Regular culture screens are carried out and antibiotics (regimens determined by local policy) are indicated in patients with any clinical suggestion of infection. Attempts to reduce infection with prophylactic antibiotics were successful but with the emergence of serious resistance problems in up to $10 \%$ of patients. Prophylactic antifungals should be considered early because of diagnostic difficulties and the high mortality associated with systemic fungal sepsis.

\section{Nutrition}

Patients with ALF are hypermetabolic. Enteral nutrition via a gastric or post-pyloric tube is the mainstay of nutritional support regimens in the absence of contraindications. Erythromycin or metoclopramide are used as pro-kinetic agents in patients with large gastric aspirates. Patients with
ALF have both peripheral and hepatological insulin resistance. The practicality of parenteral feeding was shown in a small study but is not the preferred mode of treatment because of the increased risk of sepsis.

\section{Coagulopathy}

Coagulopathy is another cardinal feature of ALF. The repletion of coagulation factors is obviously necessary for clinical bleeding and before major invasive procedures. However, the prophylactic correction of the coagulopathy, although intuitive and commonly practised, complicates the determination of prognosis and has not been shown to be of clinical benefit. Greater attention should be paid to thrombocytopaenia, with or without disseminated intravascular coagulation, which correlates much more closely with the risk of clinical bleeding than prothrombin times or INRs. The role of recombinant factor VII has not yet been established in ALF.

- An overall management strategy plan should be developed for each patient

- Coagulation derangements should not be corrected prophylactically

- Neurological, cardiovascular, and multisystem failure are the commonest causes of death

- Renal, infectious, and respiratory complications are common and contribute to mortality

- Metabolic derangements and nutrition are usually manageable

- Early and aggressive monitoring and treatment of the complications is advisable

\section{LIVER TRANSPLANTATION}

Liver transplantation plays a central part in the modern management of ALF, which now accounts for $5 \%-12 \%$ of all liver transplant activity. ${ }^{3}$ Donor organ allocation systems prioritise patients with ALF and $45 \%-50 \%$ of patients undergo transplantation. Up to $25 \%$ of patients are deemed to have contraindications to transplantation and the remainder deteriorate before on organ is allocated.

The criteria for selecting patients for liver transplantation in the UK have been outlined earlier. Alternatively, the development of encephalopathy in association with a progressive coagulopathy is a commonly used selection criterion, but one that risks unnecessary transplantation given survival rates of $39 \%-67 \%$ in some of the commoner aetiologies with medical management. Another pragmatic approach is to list all patients with ALF for transplantation and make the decision to proceed when an organ becomes available. This approach has the attraction of maximising the delivery of transplantation to this patient population, but it also increases the risk of unnecessary transplantation.

The removal of patients from the waiting list when the clinical condition deteriorates to the extent that transplantation is futile is both emotive and difficult. No definite role has yet been confirmed for specific cut off points for intracranial pressure or cerebral metabolic rates for oxygen in deciding the vexed question of when irreversible brain damage has occurred in the setting of ALF. Accelerating inotrope requirements, uncontrolled sepsis, and severe respiratory failure are other imprecise contraindications to transplantation. These contraindications are age sensitive as younger 
patients are more resilient and more likely to reverse these complications after liver transplantation.

\section{Transplant operation}

Most transplants are full organ grafts from cadaveric donors. Auxiliary liver transplantation has been performed in a small number of patients deemed to have the potential to recover normal liver function and morphology. This combines the immediate advantages of transplantation with the ability the withdraw immunosuppression and sacrifice the graft when regeneration has been seen in the native liver 6-36 months later. However, the full benefits of hepatectomy in respect to cardiovascular and neurological stability are not obtained and the native liver has not always regenerated. Outside the UK, living related donation of tissue has been used in the setting of ALF.

The repletion of coagulation factors, and platelets where necessary, before surgery adequately reverses the clinical coagulopathy in most cases, and intraoperative blood losses are remarkably low. This reflects both the poor correlation between studies of coagulation factors and the risk of surgical bleeding, and the absence of portal hypertension. Cerebral oedema may be problematic during the dissection phase and the period immediately after reperfusion but often improves dramatically during the anhepatic phase of the transplant operation. Cerebral autoregulation is restored within 48 hours of successful transplantation and monitoring of intracerebral pressure and cerebral perfusion pressure should continue during this period in patients susceptible to cerebral oedema.

The profile of sepsis, including fungal infection, seen in ALF extends into the post-transplant period and is further aggravated by immunosuppressive therapy. Renal function may improve dramatically in the immediate postoperative period but more often renal support may be necessary for many weeks after successful transplantation. The potent immunosuppressive agents and some antimicrobial drugs commonly used are potentially nephrotoxic and the use of these agents should be modified to promote the return of renal function.

\section{Results}

The overall survival rates are in the region of $60 \%-65 \%$, but individual centres have reported higher survival in the $75 \%-$ 90\% range. ${ }^{3}$ Patients receiving liver transplants for ALF (median age 28 years) are younger those undergoing elective transplantation (median age 44 years). The age of the patient influences selection for liver transplantation in ALF more than for elective transplantation. The aetiology of ALF correlated with outcome in some centres with the best results seen in transplantation for Wilson's disease and the worst for idiosyncratic drug reactions. In paracetamol induced liver failure, survival was best when the transplant was performed within four days of the overdose. This reflects the role of sepsis in propagating the continued deterioration in these patients and the inability of liver transplantation to reverse this complication.

The survival rates also decrease with progression through the grades of encephalopathy at the time of transplantation; in one series the figures were $90 \%$ for grade $1,77 \%$ for grade $2,79 \%$ for grade 3, and $54 \%$ for grade 4 . As with liver transplantation in general, renal function correlated with outcome and serum creatinine concentrations above $200 \mu \mathrm{mol} / \mathrm{l}$ were associated with a poorer outcome. Acidosis and Apache III scores at the time of transplantation were good indicators of the severity of the illness and also correlated with outcome. However, none of these parameters were sufficiently discriminatory to use as criteria to disqualifying individual cases from transplantation.
- ALF accounts for up to $12 \%$ of liver transplant activity

- Nearly half of ALF patients have liver transplants (excluding paracetamol patients)

- Survival rates are generally in the $75 \%-90 \%$ range

- Auxiliary transplants are an exciting alternative offering the possibility of ultimate return to transplant free survival

- Living related transplantation is being increasingly used although not yet in the UK

\section{Liver support systems}

The search for artificial liver support systems to manage patients with ALF has been unrewarding to date. The performance of the devices has been variable but has been better at "bridging" patients to transplantation than at improving transplant free survival. The systems can be divided into bio-artificial livers using cell based therapies and dialysis based methods or plasmapheresis.

Historically, charcoal haemoperfusion generated considerable interest and the initial enthusiasm emanated from an uncontrolled study incorporating 75 patients in which a significant survival benefit in the treated patients as compared with historical controls was found (65\% compared with $15 \%) .{ }^{15}$ However, a subsequent controlled study failed to show a survival benefit in the active limb. ${ }^{16}$

Two cell based devices have been evaluated within the past decade. One used up to 50 grams of porcine hepatocytes and preliminary data were encouraging with respect to a number of relevant biological variables. However, a randomised controlled trial enrolling 147 patients with ALF and 27 patients with primary graft non-function found no overall difference in 30 day survival rates. ${ }^{17}$ On subset analysis, there did seem to be a significant benefit in terms of survival for paracetamol induced ALF and those patients who developed encephalopathy within two weeks of jaundice. Another system incorporated hepatocytes in the form of hepatoblastoma cells $\left(\mathrm{C}_{3} \mathrm{~A}\right.$ cell line) that were grown to confluence in the extra-capillary compartment of a hollow fibre filter. ${ }^{18}$ Once again preliminary data were encouraging but clinical trails have not yet progressed beyond the phase 2 stage.

Liver support systems that do not use biological components include albumin dialysis (MARS) and plasmapheresis. Albumin dialysis has been advocated for the removal of albumin bound toxins and is complemented by standard dialysis removing water soluble toxins. Data on patients with ALF are limited and to date unconvincing. Plasmapheresis protocols have used membrane filtration with a pore size of $0.65 \mathrm{~mm}$ and exchanged $15 \%$ of body weight on up to three consecutive occasions. Improvement in encephalopathy, cerebral blood flow, and cerebral perfusion pressure has been reported without any deleterious change in intra-cranial pressure. ${ }^{19}$ Mean arterial pressure improved significantly with an improvement in systemic vascular resistance. However, once again there is as yet a lack of controlled clinical data.

- Liver support systems remain a holy grail despite recent enthusiasm

- Biological and non-biological systems have been evaluated

- No convincing phase three trial has shown efficacy of these devices 


\section{MULTIPLE CHOICE QUESTIONS (TRUE (T)/FALSE (F); ANSWERS AT END OF REFERENCES)}

1. Acute liver failure (ALF) is:

(A) diagnosed when a coagulopathy complicates an acute liver injury

(B) commonly caused by alcohol

(C) becoming less common after paracetamol overdose

(D) rarely caused by hepatitis $\mathrm{C}$

(E) often caused by hepatitis E in Africa

2. The following tests are routine in the investigation of the aetiology of ALF:
(A) IgG anti-HAV
(B) HBV DNA
(C) urinary copper
(D) ultrasound
(E) liver biopsy

3. The following tests are relevant in the assessment of prognosis of ALF:

(A) prothrombin time or INR

(B) serum lactate

(C) serum transaminases

(D) ultrasound

(E) liver biopsy

4. The following are recognised complications of ALF:
(A) cerebral oedema
(B) acute lung injury
(C) hypoadrenalism
(D) hypoglycaemia
(E) hypothermia

5. Liver transplantation in the management of ALF:

(A) is used in up to half of cases

(B) accounts for $25 \%$ of transplant activity

(C) yields survival rates consistently over $80 \%$

(D) may be a temporary therapy
(E) is contraindicated in patients with intracranial pressure $>30 \mathrm{~mm} \mathrm{Hg}$

\section{REFERENCES}

1 O'Grady JG, Schalm SW, Williams R. Acute liver failure: redefining the syndromes. Lancet 1993;342:273-5.

2 Bernal B. Changing patterns of causation and the use of transplantation in the United Kingdom. Semin Liver Dis 2003;23:227-37.

3 O'Grady J. Acute liver failure. In: O'Grady J, Lake J, Howdle P, eds. Comprehensive clinical hepatology, 1st ed. London: Mosby, 2000:30.1-20.

4 O'Grady JG, Alexander GJ, Hallyar KM, et al. Early indicators of prognosis in fulminant hepatic failure. Gastroenterology 1989;97:439-45.

5 O'Grady J, Alexander G, Hallyar K, et al. Early indicators of prognosis in fulminant hepatic failure. Gastroenterology 1989;97:439-45.

6 Bernal W, Donaldson N, Wyncoll D, et al. Blood lactate as an early indicator of outcome in paracetamol-induced acute liver failure. Lancet 2002;359:558-63.

7 Strauss GI, Knudsen GM, Kondrup J, et al. Cerebral metabolism of ammonia and amino acids in patients with fulminant hepatic failure. Gastroenterology 2001;121:1109-19.

8 Blei AT, Olafsson S, Webster S, et al. Complications of intracranial pressure monitoring in fulminant hepatic failure. Lancet 1993:341:157-8.

9 Keays RT, Alexander GJ, Williams R. The safety and value of extradural intracranial pressure monitors in fulminant hepatic failure. J Hepatol 1993; 18:205-9.

10 Jalan R, Damink SW, Deutz NE, et al. Moderate hypothermia for uncontrolled intracranial hypertension in acute liver failure. Lancet 1992:354:1164-8.

11 Murphy N, Auzinger G, Bernel W, et al. The effect of hypertonic sodium chloride on intracranial pressure in patients with acute liver failure. Hepatology 2004;39:464-70.

12 Harry R, Auzinger G, Wendon J. The clinical importance of adrenal insufficiency in acute hepatic dysfunction. Hepatology 2002;36:395-402.

13 Davenport A. The management of renal failure in patients at risk of cerebral oedema/hypoxia. New Horiz 1995;3:717-24.

14 Rolando N, Wade J, Davalos $M$, et al. The systemic inflammatory response syndrome in acute liver failure. Hepatology 2000;32:734-9.

15 Gimson AE, Braude S, Mellon PJ, et al. Earlier charcoal haemoperfusion in fulminant hepatic failure. Lancet 1982;ii:681-3.

16 O'Grady JG, Gimson AE, O'Brien CJ, et al. Controlled trials of charcoal hemoperfusion and prognostic factors in fulminant hepatic failure. Gastroenterology 1988;94:1186-92.

17 Chen SC, Mullon C, Kahaku E, et al. Treatment of severe liver failure with a bioartificial liver. Ann N Y Acad Sci 1997;831:350-60.

18 Ellis AJ, Hughes RD, Wendon JA, et al. Pilot-controlled trial of the extracorporeal liver assist device in acute liver failure. Hepatology 1996:24:1446-51.

19 Clemmesen JO, Larsen FS, Ejlersen E, et al. Haemodynamic changes after high-volume plasmapheresis in patients with chronic and acute liver failure. Eur J Gastroenterol Hepatol 1997;9:55-60.

\section{ANSWERS}

1. (A) F, (B) F, (C) T, (D) T, (E), F; 2. (A) F, (B) T, (C), F, (D), F, (E), F; 3. (A) T, (B) T, (C) F, (D) T, (E), F; 4. (A), T, (B) T, (C) T, (D), T, (E) F; 5. (A) T, (B) F, (C) F, (D) T, (E) F. 